MODELING, IDENTIFICATION AND CONTROL, 2003, vOL. 24, No. 4, 217-229

doi:10.4173/mic.2003.4.3

\title{
Adaptive Tracking Control of an Electro-Pneumatic Clutch Actuator
}

\author{
GLENN-OLE KAASA* and MASANORI TAKAHASHI $\dagger$
}

Keywords: adaptive tracking control, high-gain feedback, electro-pneumatic clutch actuation

\begin{abstract}
This paper deals with the application of a simple adaptive algorithm for robust tracking control of an electro-pneumatic clutch actuator with output feedback. We present a mathematical model of the strongly nonlinear system, and implement an adaptive algorithm, based on a parallel feedforward compensator (PFC) to remove the relative-degree-I restriction. We propose a practical method of constructing the PFC, and introduce a simple modification that removes an inherent restriction on bandwidth of the nonlinear system. We show that the adaptive algorithm deals well with nonlinearities, and we achieve tracking corresponding to a settling-time of $150 \mathrm{~ms}$.
\end{abstract}

\section{Introduction}

Electro-pneumatic clutch actuators are used on heavy-duty vehicles as part of a gear shift system for automating manual transmissions. A main goal is to have a control system for the pneumatic actuator which is robust to a wide range of different clutch characteristics. This permits that the same servo can be mounted on a wide range of different clutch types without the need of individual tuning of the controller parameters, or change of controller structure.

Only a few published papers are found that address the control of electropneumatic clutch actuation, see e.g. (Tanaka \& Wada, 1995), (Kaasa, 1999) and (Kaasa et al., 2001).

This paper deals with the application of a simple direct adaptive algorithm for robust tracking control of an electro-pneumatic clutch actuator with output-feedback. We present a mathematical model of the strongly nonlinear system, and implement an adaptive algorithm, based on a parallel feedforward compensator (PFC) to remove the relative-degree-1 restriction. We propose a practical method of constructing the PFC, and introduce a simple modification that removes an inherent restriction on bandwidth of the nonlinear system. The performance and robustness of the proposed controller is tested by simulations on the nonlinear model.

The paper is organized as follows: In Section 1, a description of the clutch actuator system is given, with a brief outline of the derivation of the mathematical models used for simulation and controller synthesis. Section 2 deals with the adaptive tracking design, and in Section 3, the adaptive controller is implemented and tested by simulations. Finally, some conclusions are drawn in Section 4.

This work was supported in part by a research grant from the U.S.- Norway Fulbright Foundation, and in part by a research grant from Kongsberg Automotive ASA, and the Norwegian Research Council, and was presented at the int. symposium on Advanced Control of Industrial Processes, Kumamoto University, Japan, June 10-11 2002. (http:// www.adconip.org)

*Norwegian University of Science and Technology (NTNU), N-7491 Trondheim, Norway, glenn-ole@kaasa.as

†Ariake National College of Technology, 150 Higashi-Hagio, Omuta, Fukuoka, 836 8585, Japan, taka@ariake-nct.ac.jp 


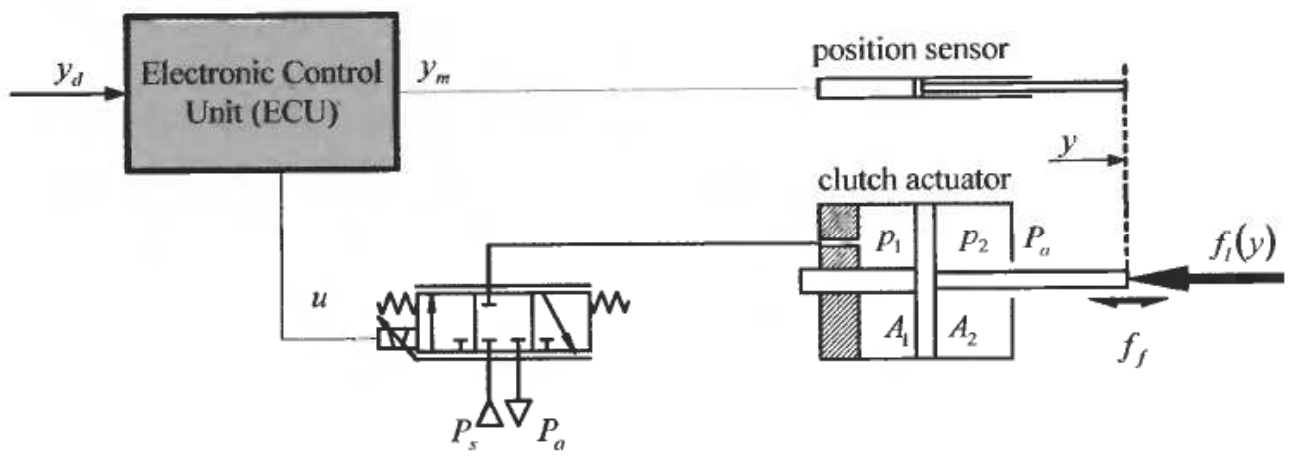

Figure 1. Simple schematic of the clutch actuator system.

\section{Clutch actuator system}

\subsection{System description}

A simplified schematic of the clutch actuator system is given in Figure 1. The actuator position $y$ is controlled by operating the proportional valve to produce the necessary pressure $p_{1}$ acting against the piston to balance the load force $f_{l}$, and compensate for the friction forces $f_{f}$. The back-chamber $\left(p_{2}\right)$ is connected to atmosphere through a restriction. Some actuators have a small restriction such that actuator movements are causing a dynamic back-pressure $p_{2}$.

The servo actuator is used to operate the clutch disc position during gear shift. The servo has to perform the tracking of a position demand signal $y_{d}$, and the tracking performance must be robust to a wide range of variations in the system and its operating conditions; e.g. in supply pressure $P_{s}$, the cylinder wall temperature $T_{w}$, and changes (drift) in the clutch spring force characteristic $f_{l}(y)$ due to ageing and wear of the clutch. The controller must also eliminate the effect of the relatively high friction forces $f_{f}$ in the system.

\subsection{Mathematical model}

The mathematical equations that constitutes the nonlinear dynamic model of the clutch actuator are summarized below. The full model is presented in a recursive order of derivation: (i) The output of interest is $y$, and it is governed by the motion dynamics where the pressures $p_{1}$ and $p_{2}$ are considered the inputs, (ii) the pressures are expressed by the pressure and temperature dynamics which is controlled by the valve opening $a_{v}$, and finally, (iii) the valve dynamics has our available control $u$ as input.

(i) Motion dynamics. The motion dynamics of the actuator piston is expressed by Newton's 2nd law of motion. The resulting force acting on the actuator piston is composed of the load force $f_{l}$, the resulting friction force $f_{f}$, and the actuator pressure force $\left(A_{1} p_{1}-A_{2} p_{2}\right)$, giving

$$
M \frac{d^{2} y}{d t^{2}}=A_{1} p_{1}-A_{2} p_{2}-f_{l}-f_{f}
$$

Here, $y, p_{1}, p_{2}$ are the piston position and pressures, where indices 1 and 2 are referring to chamber 1 and chamber 2, respectively; $M$ is the effective mass of moving parts, and $A_{1}$ and $A_{2}$ are the effective piston areas. 

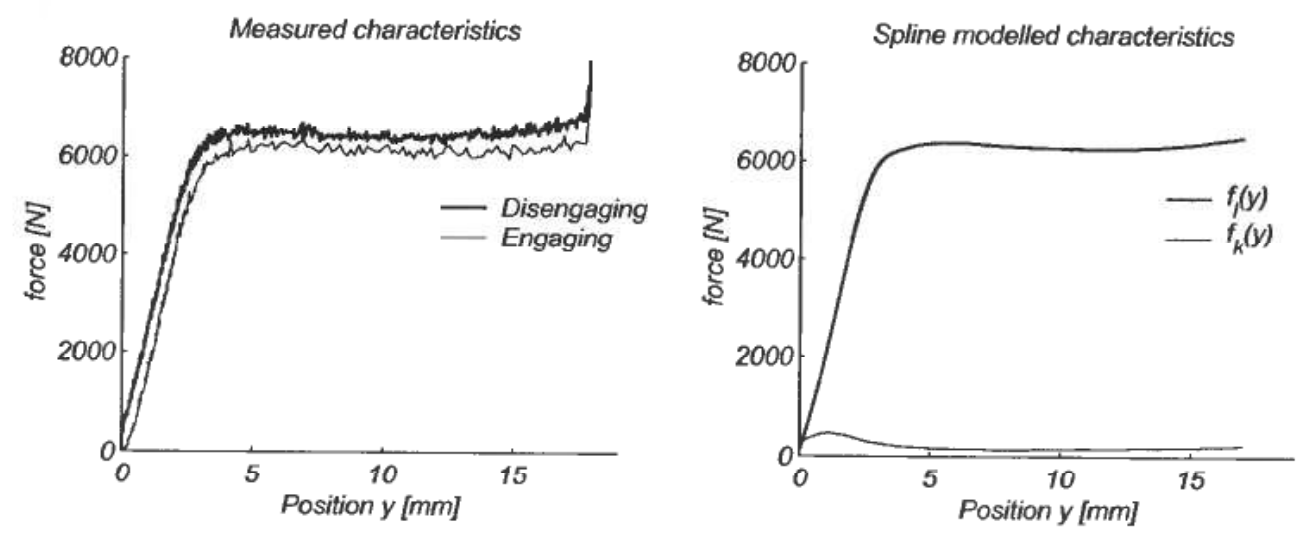

Figure 2. The left plot shows the measured quasi-static load characteristics of the clutch actuator. The right plot shows the extracted mean load characteristic $f_{l}(y)$, and the kinetic dry friction characteristic $f_{k}(y)$.

Load force: $f_{l}$ is a stiff and highly nonlinear spring force which is caused by the clutch compression spring and a counteracting, much weaker actuator spring. A resulting quasi-static ${ }^{1}$ load characteristic is obtained from measurements (Figure 2, left) and a mean load characteristic is modelled as $f_{l}(y)$ (Figure 2, right).

Friction force: The mechanical friction is composed of static and kinetic dry friction, and viscous friction. The hysteresis in the measured load characteristic is caused by the kinetic dry friction force, denoted $f_{k}$. The kinetic dry friction characteristic $f_{k}(y)$ is obtained from the measured load characteristic (Figure 2). A simplified model of the total resulting friction force is given by

$$
f_{f}(\cdot)=D_{v} \dot{y}+\left\{\begin{array}{cc}
f_{k} \operatorname{sgn}(\dot{y}) & \dot{y} \neq 0 \\
\min \left(\left|f_{\Sigma}\right|, f_{k}\right) \operatorname{sgn}\left(f_{\Sigma}\right) & \dot{y}=0
\end{array}\right.
$$

where $D_{v}$ is the viscous friction coefficient, and $f_{\Sigma} \triangleq A_{1} p_{1}-A_{2} p_{2}-f_{l}$ is denoting the sum of forces acting on the actuator, except friction.

(ii) Pressure dynamics. The derivation of the pressure and temperature dynamics of the pneumatic chambers are based on simple thermodynamics; the following assumptions are made:

(A1) At the attainable pressures, air may be treated as a perfect gas which obeys the ideal gas law with negligible error.

(A2) The dimensions of the system are relatively small, hence, it is reasonable to assume that the thermodynamic states are uniform within the control volume.

(A3) Flow loss due to friction is negligible, thus the flow through pipes is isentropic.

(A4) Energy change in the fluid due to elevation is negligible.

(A5) The specific heats $c_{p}$ and $c_{v}$ of air at the attainable pressures and temperatures are assumed to be constant, i.e. not functions of pressure and temperature.

${ }^{1}$ The load characteristics is obtained by one slow disengagement and subsequent engagement of the clutch, thus the denotation quasi-static. 
Remark 1. Note that a usual approximation is to neglect the heat transfer between the fluid and the cylinder wall, that is, $\dot{Q}=0$. However, for the clutch application, the generation of heat near the cylinder can be very high, thus the effect of heat transfer may be significant.

The pressure and temperature dynamics in chamber $j=1,2$ are given by

$$
\begin{aligned}
\frac{d p_{j}}{d t}=\frac{\kappa \dot{V}_{j}}{V_{j}} p_{j}+\frac{\kappa R T_{\text {jin }}}{V_{j}} w_{j i n}-\frac{\kappa R T_{j}}{V_{j}} w_{\text {jout }}+\frac{(\kappa-1)}{V_{j}} H_{w} A_{w j}\left(T_{w}-T_{j}\right) \\
\frac{d T_{j}}{d t}=-\frac{(\kappa-1) \dot{V}_{j}}{V_{j}} T_{j}+\frac{\left(\kappa T_{j i n}-T_{j}\right) R T_{j}}{p_{j} V_{j}} w_{j i n}-\frac{(\kappa-1) R T_{j}^{2}}{p_{j} V_{j}} w_{j o u t} \\
+\frac{(\kappa-1) T_{j}}{p_{j}} H_{w} A_{w j}\left(T_{w}-T_{j}\right)
\end{aligned}
$$

where the functions $V_{j}, A_{j w}, w_{j i n}$, and $w_{j o u t}$ are the chamber volume, effective heat transfer area, inlet flow, and outlet flow, respectively (all addressed in the subsequent paragraphs); the parameters $\kappa, R, H_{w}$, and $T_{w}$ are the ratio of specific heats, gas constant of air, convective heat coefficient and cylinder wall temperature.

The volumes of the two chambers are given by the functions

$$
V_{1}(y)=V_{01}+A_{1} y, \quad V_{2}(y)=V_{02}-A_{2} y
$$

where $V_{0 j}, j=1,2$ are the volumes when $y=0$. Furthermore, the effective areas of heat transfer are given by the functions

$$
\begin{aligned}
& A_{w 1}=A_{e w}+A_{c w} \frac{y}{y_{\max }} \\
& A_{w 2}=A_{e w}+A_{c w}\left(1-\frac{y}{y_{\max }}\right)
\end{aligned}
$$

where $A_{e w}$ and $A_{c w}$ are the effective end cap and cylinder wall surfaces, and $y \in\left[0, y_{\max }\right]$ is the operating range of the cylinder.

Flow-rate model: The mass flow-rate $w$ of air through a variable restriction is modelled as

$$
w\left(a_{r}, p_{h}, T_{h}, p_{l}\right)=\sqrt{\frac{T_{n}}{T_{h}}} \rho_{n} \frac{C_{r}}{a_{r \max }} a_{r} p_{h} \Omega
$$

where $\Omega$ is a function of the pressure ratio over the restriction, and is given by

$$
\Omega\left(p_{h}, p_{l}\right) \triangleq \frac{w}{w^{*}}=\left\{\begin{array}{cl}
1 & \frac{p_{l}}{p_{h}} \leqslant B \\
\left.\sqrt{1-\left(\frac{p_{l}}{p_{h}}-B\right.}\right)^{2} & \frac{p_{l}}{p_{h}}>B
\end{array}\right.
$$

Here $a_{r}, p_{h}, T_{h}$, and $p_{l}$ are the restriction area, upstream (high) pressure and temperature, and downstream (low) pressure; the parameters $\rho_{n}, T_{n}$ are the density 


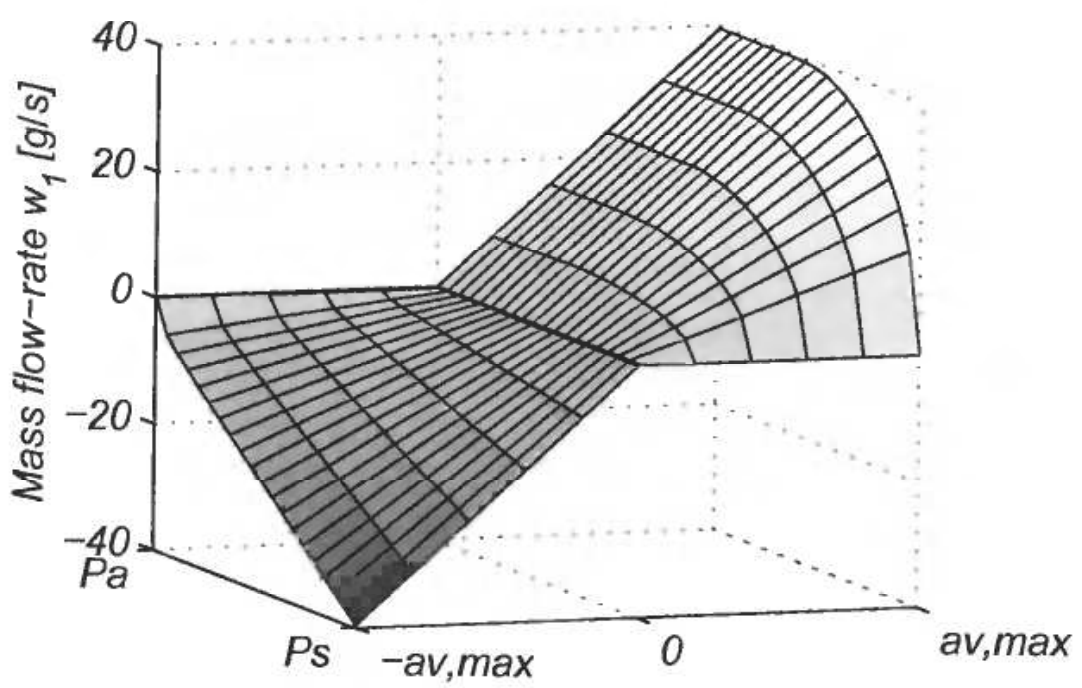

Figure 3. Steady-state flow characteristics of the pneumatic proportional valve.

and temperature at the defined normal state of air; $C_{r}$ is the sonic conductance at maximum opening $a_{r \max }$, and $B=\left(p_{l} / p_{h}\right)^{*}$ is the sonic pressure ratio of the restriction ${ }^{2}$.

To clearly state the complete flow-rate model of the valve $\left(w_{1}\right)$, we express the model as

$$
w_{1}=\left\{\begin{array}{cc}
w_{\text {in }}\left(a_{v}, P_{s}, T_{s}, p_{1}\right) & P_{s} \geqslant p_{1} \\
-w_{\text {out }}\left(a_{v}, p_{1}, T_{1}, P_{s}\right) & P_{s}<p_{1}
\end{array}\right\} a_{v} \geqslant 0
$$

The valve flow characteristic for chamber pressure $p_{1} \in\left[P_{a}, P_{s}\right]$ and valve opening $a_{v} \in\left[-a_{v \max }, a_{v \max }\right]$ is plotted in Figure 3. The flow through the outlet restriction of chamber 2 is given by

$$
w_{2}=\left\{\begin{array}{cc}
w_{\text {in }}\left(A_{r}, P_{a}, T_{a}, p_{2}\right) & P_{a} \geqslant p_{2} \\
-w_{\text {out }}\left(A_{r}, p_{2}, T_{2}, P_{a}\right) & P_{a}<p_{2}
\end{array}\right.
$$

where $A_{r}$ is the restriction area.

${ }^{2}$ The superscript asterix denotes sonic condition. For pressure ratios below sonic, i.e.

$$
\frac{p_{l}}{p_{h}} \leqslant\left(\frac{p_{l}}{p_{h}}\right)^{*}
$$

the flow is said to be sonic, or choked. Sonic, because the velocity in the smallest nozzle area then equals the speed of sound, and choked, because further lowering of the downstream pressure $p_{l}$ does not cause an increase in the mass flow-rate $w$. 
(iii) Valve dynamics. The dynamics of the spool actuator of the valve is approximated by the first-order linear dynamic model

$$
\frac{d a_{v}}{d t}=-\frac{1}{\tau_{v}} a_{v}+\frac{K_{v}}{\tau_{v}} u
$$

where $a_{v} \in\left[-a_{v \max }, v_{\max }\right]$ and $u \in[-10,10]$ are the valve opening, and control input; $\tau_{v}$ and $K_{v}$ are the time-constant, and the steady-state gain from $u$ to $a_{v}$.

\subsection{Design model}

The full nonlinear model is of order $n=7$, with the states $\mathrm{z}=\left[y, \dot{y}, p_{1}, p_{2}, T_{1}\right.$, $\left.T_{2}, a_{v}\right]^{T}$ and relative degree $\rho=4$. In anticipation of the adaptive control design, we note that the full nonlinear model can be expressed on the form

$$
\begin{aligned}
& \dot{\mathbf{x}}=\mathbf{A x}+\mathbf{b} u+\mathbf{d}(\mathbf{x}, \xi, u) \quad y=\mathbf{c}^{T} \mathbf{x} \\
& \dot{\xi}=\boldsymbol{\phi}(\mathbf{x}, \xi, u)
\end{aligned}
$$

where the first equation is the dynamics of the $\rho$ observable states, $\mathbf{x}=\left[y, \dot{y}, p_{1}, a_{v}\right]^{T}$, and $\phi(\mathbf{x}, \xi, u)$ is the dynamics of the remaining non-observable states, $\xi=\left[T_{1}, p_{2}, T_{2}\right]^{T}$. We can show that the zero-dynamics (14) is stable, thus the system is minimumphase. We can also show that the nonlinear part $\mathbf{d}(\mathbf{x}, \xi, u)$ in (13) is bounded when $\mathbf{x}$, $\xi$, and $u$ are bounded.

The derivation of the linear model used for control design is obtained through simplifications of the full nonlinear model. The steps are briefly summarized below:

(i) We neglect the dynamics in $p_{2}$, that is, $\dot{p}_{2} \approx 0 \Rightarrow p_{2}=P_{a}$.

(ii) The pressure and temperature dynamics are coupled, and can be reasonably approximated by a polytropic process;

$$
T_{j}=T_{0}\left(\frac{P_{0}}{p_{j}}\right)^{(1-\gamma / y)} \quad j=1,2 \quad \gamma \in[1, \kappa]
$$

(iii) When linearizing we must also neglect the dry friction force in the friction model (2).

The simplified model is now of order $\rho$, with the states $\mathbf{x}=\left[y, \dot{y}, p_{1}, a_{v}\right]^{T}$. A simple linear model used for design is then obtained by linearization along the steady-state solution $\mathbf{x}^{*}=\left[y^{*}, 0, p_{1}^{*}, 0\right]^{T}$. This model can be represented by the transfer function

$$
G(s)=\frac{K \omega}{\left(\tau_{v} s+1\right)\left(s^{2}+2 \zeta \omega s+\omega^{2}\right) s}
$$

where the parameters $K, \zeta$, and $\omega$ are the high frequency gain, damping coefficient, and resonance frequency, respectively. Table 1 lists values for three typical operating points $y^{*}$, and gives a notion of the strong nonlinearities in the system.

Table 1. Parameters at $y^{*}$

\begin{tabular}{lcccc}
\hline$y^{*}$ & $\mathrm{~mm}$ & 2.0 & 4.0 & 8.0 \\
\hline$K$ & {$[\mathrm{~V} / \mathrm{mm}]$} & 89 & 260 & 525 \\
$\zeta$ & - & 0.02 & 0.04 & 0.05 \\
$\omega$ & {$[\mathrm{rad} / \mathrm{s}]$} & 172 & 81 & 64 \\
\hline
\end{tabular}




\section{Adaptive tracking design}

\section{Basic algorithm}

First, we review the basic design method of an adaptive algorithm with a dead zone (Peterson \& Narendra, 1982). Consider a linear plant

$$
\dot{\mathbf{x}}=\mathbf{A x}+\mathbf{b} u+\mathbf{d} \quad y=\mathbf{c}^{T} \mathbf{x}
$$

where $\mathbf{x} \in \mathbb{R}^{n}, u \in \mathbb{R}, y \in \mathbb{R}$, and $\mathbf{d} \in \mathbb{R}^{n}$ are the state vector, the control input, the output, and a disturbance vector, respectively. Without loss of generality, assume that the plant is controllable and observable. Furthermore, assume that the disturbance $\mathbf{d}$ is bounded by a finite, positive constant $\rho_{d}$;

$$
\|\mathbf{d}\|_{\infty} \leqslant \rho_{\mathbf{d}}
$$

Neglecting the disturbance, the plant (17) may be represented by the transfer function $G(s)=\mathbf{c}^{T}(s \mathbf{I}-\mathbf{A})^{-1} \mathbf{b}$.

The control objective of the adaptive tracking controller is to make the plant output $y(t)$ track the output $y_{m}(t)$ of a reference model given by

$$
\dot{\mathbf{x}}_{m}=\mathbf{A}_{m} \mathbf{x}_{m}+\mathbf{b}_{m} r \quad y_{m}=\mathbf{c}_{m}^{T} \mathbf{x}_{m}
$$

where $\mathbf{x}_{m} \in \mathbb{R}^{n_{m}}, u_{m} \in \mathbb{R}, y_{m} \in \mathbb{R}$, and $y_{m} \in \mathbb{R}$. The model is constructed such that the order $n_{m} \leqslant n$, and all signals are bounded by a positive constant $\rho_{m}$;

$$
\max \left(\left\|x_{m}\right\|_{\infty},\left\|\left[y_{m}, \dot{y}_{m}\right]^{T}\right\|_{\infty}\right) \leqslant \rho_{m}
$$

The reference model (19) can be represented by the transfer function $G_{m}(s)=\mathbf{c}_{m}^{T}\left(s \mathbf{I}-\mathbf{A}_{m}\right)^{-\mathbf{1}} \mathbf{b}_{m}$.

Assume that the plant (17) satisfies Condition A:

\section{Condition A:}

(C1) The plant is a minimum-phase system.

(C2) The relative degree is one.

(C3) The high-frequency gain is positive.

Then, the adaptive tracking controller is given by the control law

$$
u=-k e \quad e \triangleq y-y_{m}
$$

and the update law

$$
\dot{k}=\gamma e^{2} \Delta(e) \quad \Delta(e)= \begin{cases}1 & |e| \geqslant \delta_{0} \\ 0 & |e|<\delta_{0}\end{cases}
$$

Here, $e$ is the tracking error, $\gamma$ is the adaption gain, and $\delta_{0}$ is a small, positive constant which determines the level of tracking precision. For the above adaptive controller, with the initial gain $k_{0}=k\left(t_{0}\right)$ bounded, the following stability and convergence properties can be proved (Takahashi et al., 2001):

Theorem 2. Suppose that the plant (17) satisfies Condition A. Then, all of the signals of the adaptive tracking system (21) and (22) are bounded, and $\lim _{t \rightarrow \infty}|e(t)| \leqslant \delta_{0}$, despite the existence of bounded disturbances that satisfy (18).

Proof. See Takahashi et al., 2001. 


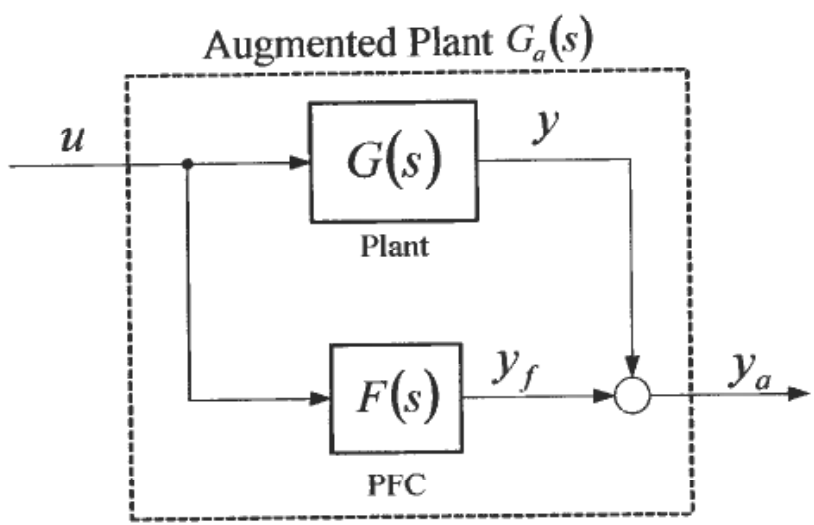

Figure 4. Block diagram of the augmented plant $G_{a}(s)$, which is constructed by augmenting the plant $G(s)$ by $F(s)$.

\subsection{A modified algorithm}

For plants with relative degree higher than 1 , a parallel feedforward compensator (PFC) is constructed such that an augmented plant $G_{a}(s)$ satisfies Condition $\mathrm{A}$, see Figure 4.

However, the introduced PFC causes an offset in the output, such that the convergence property $\lim _{t \rightarrow \infty}|e| \leqslant \delta_{0}$ is not longer achieved. The remedy is to modify the adaptive scheme with an additional feedback loop to counteract the bias effect of the PFC. The modified adaptive tracking algorithm is given by

$$
\begin{aligned}
& u=-k e_{a} \quad e_{a} \triangleq y+y_{f}-\tilde{y}_{m} \\
& \dot{k}=\gamma e_{a}^{2} \Delta\left(e_{a}\right)
\end{aligned}
$$

where the PFC output $y_{f}$, and the modified reference model output $\tilde{y}_{m}$, are given by

$$
\begin{aligned}
& y_{f}=F(s) u \\
& \tilde{y}_{m}=G_{m}(s)\left(r+G_{m}(0)^{-1} y_{f}\right)
\end{aligned}
$$

A block diagram of the modified algorithm is shown in Figure 5.

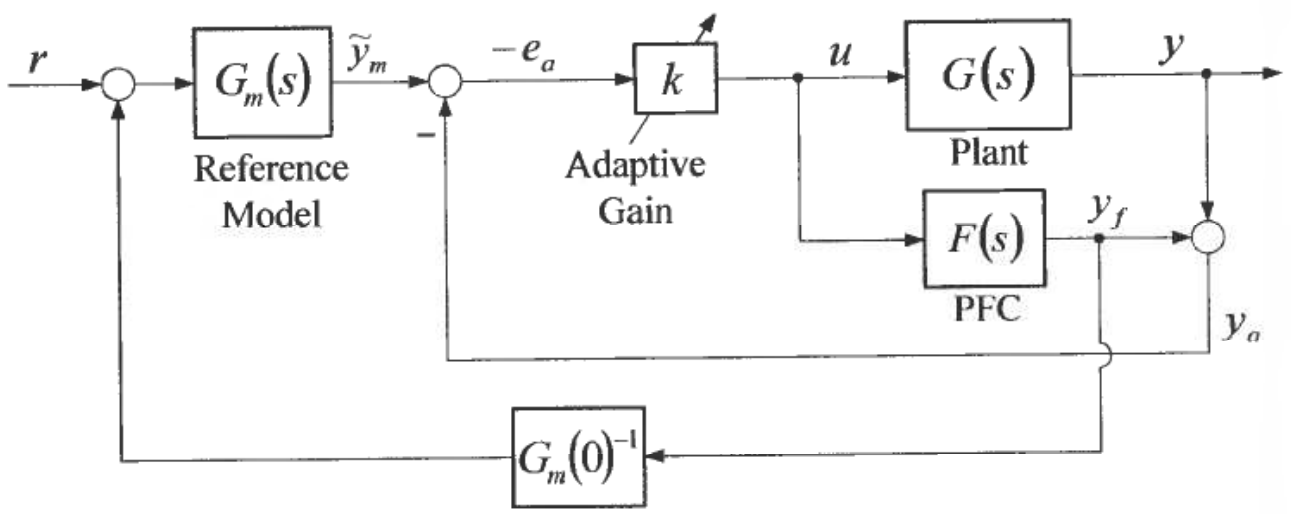

Figure 5. A modified adaptive tracking system with the PFC, $F(s)$. 
Suppose that the augmented plant

$$
\tilde{G}_{a}(s)=\frac{G(s)}{1-G_{m}(s) G_{m}(0)^{-1}}+F(s)
$$

fulfills Condition A. Then, from Theorem 2 we can verify that all the signals in the adaptive tracking system are bounded, and $\lim _{t \rightarrow \infty}\left|e_{a}(t)\right| \leqslant \delta_{0}$, despite the existence of the bounded disturbance d (Takahashi et al., 2001). Furthermore, the output of the PFC converges to zero, that is, $e_{a}(t) \rightarrow e(t)$ as $t \rightarrow \infty$.

\subsection{Construction of the PFC}

In this subsection, we propose a new practical method for constructing the PFC for arbitrary minimum-phase systems.

Consider the minimum-phase system $G(s)$, with relative degree $\rho>1$. Then, the PFC can be constructed as

$$
\begin{aligned}
F(s) & =\frac{n_{f}(s)}{d_{f}(s)} H(s)^{-1} \\
H(s) & =\kappa^{2 \rho-1} \frac{(s+1)^{\rho-1}}{\prod_{i=1}^{\rho-1}\left(s+\kappa^{2 i}\right)}
\end{aligned}
$$

where $n_{f}(s)$ and $d_{f}(s)$ are stable polynomials, and $\operatorname{deg} d_{f}(s)-\operatorname{deg} n_{f}(s)=1$.

Theorem 3. For sufficiently large $\kappa$, the PFC, $F(s)$ given by (28) and (29) makes the augmented plant, $G_{a}(s)=G(s)+F(s)$ satisfy Condition A.

Proof. First, consider the following system $G_{v}(s)$

$$
G_{v}(s)=G(s) \frac{d_{f}(s)}{n_{f}(s)}
$$

This system is minimum-phase, and its relative degree is $\rho-1$. According to (Miller \& Davidson, 1991), there exists a positive, bounded constant $\kappa_{0}$ such that for $\forall \kappa>\kappa_{0}$, the closed-loop system

$$
G_{c l}(s)=\frac{G_{v}(s)}{1+G_{v}(s) H(s)}
$$

is stabilized by the compensator $H(s)$. Since the zeros of $G_{a}(s)$ are equivalent to the poles of $G_{c l}(s)$, condition $\mathrm{A} 1$ holds. Furthermore, condition $\mathrm{A} 2$ also holds because the relative degree of $F(s)$ is one. Thus, the augmented plant $G_{a}(s)$ satisfies Condition A for $\forall \kappa>\kappa_{0}$.

Remark 4. If the plant is a minimum-phase system and the reference model is stable, then the PFC can always be constructed by the proposed method. Note also that the PFC represents a straightforward appoarch to expand the traditional model reference adaptive control algorithms (MRAC) to minimum-phase systems of arbitrary relative degree. 
The advantage in practical use of the proposal is that the design method has only one parameter $\kappa$, to be tuned. Note that when $G(s)$ is obtained by linearization about an equilibrium state $\mathbf{x}^{*}$, the constant $\kappa_{0}$ will wary with the chosen equilibrium $\mathbf{x}^{*}$. Hence, specify $\kappa_{0}^{+}=\max \kappa_{0}\left(\mathbf{x}^{*}\right), \forall \mathbf{x}^{*} \in \Omega_{x}$, where the set $\Omega_{x}$ contains all possible $\mathbf{x}^{*}$. Then, for $\kappa>\kappa_{0}^{+}$, the PFC makes the augmented plant (27) fulfill Condition A over the entire operating range of the system.

\subsection{Peak-cutting modification}

A given disturbance bound $\rho_{d}(18)$, results in a bound $\rho_{m}(20)$ on the reference model. However, this is a conservative estimate of the stability limit of the adaptive algorithm. By exploiting the properties of a given reference model, we can replace the bound $(20)$ by the less conservative stability limit

$$
\tau_{m} \geqslant \tau_{m \min } \quad\|r(t)\|_{\infty} \leqslant r_{\max }
$$

where $r_{\max }$ is the maximum reference input, and $\tau_{m \min }$ represents the minimum timeconstant where stability of the adaptive algorithm is guaranteed.

From (32) we see that the bandwidth of the adaptive system is restricted by $\rho_{d}$, and the worst-case reference input. This constraint can be removed by allowing adaption only for the low-frequency content of the reference input $r(t)$. The achievement is that the bound on reference model bandwidth is removed, at the cost of allowing tracking error for high-frequency inputs.

This modification is obtained by expanding the dead-zone of the update-law (24) according to

$$
\Delta\left(e_{a}, \mathbf{x}_{m}\right)= \begin{cases}1 & |e| \geqslant \delta_{0}, \mathbf{x}_{m} \in \Omega_{m} \\ 0 & \text { otherwise }\end{cases}
$$

where $\Omega_{m}$ is a set in which stability is assured. The bound (20) suggests the simple choice $\Omega_{m}=\left\{\forall \mathbf{x}_{m} \|\left.\mathbf{x}_{m}\right|_{\infty}<\rho_{m}\right\}$. Again, this is a conservative restriction which is improved by exploiting the properties of the reference model. A better choice is to use $\Omega_{m}=\left\{\left.\forall \mathbf{x}_{m}|| \overline{\mathbf{x}}_{m}\right|_{\infty}<\rho_{m}\right\}$, where in $\overline{\mathbf{x}}_{m}$ the states are weighted by $\tau \geqslant \tau_{m \text { min }}$ according to $\bar{x}_{m, i}=\tau^{i-1} x_{m, i}, i=1, \ldots, 4$.

\subsection{Leakage modification}

To preserve robustness in the case of unexpected disturbances ${ }^{3}$ that exceeds the assumed bound $\rho_{d}$, we introduce an additional leakage term in our adaptive controller, given by

$$
\dot{k}=\left(\gamma e_{a}^{2}-\sigma k^{2}\right) \Delta\left(e_{a}, \mathbf{x}_{m}\right) \quad \sigma=\frac{\gamma \delta_{0}^{2}}{k_{+}}
$$

The introduced leakage will provide us with an upper bound on $k(t)$ near $k_{+}$, but will not effect the adaption for low $k(t)$. The proposed modification is adapted from a class of modifications which is discussed in the survey paper by loannou \& Datta (1991).

\footnotetext{
${ }^{3}$ A such disturbance can be the worst-case scenario where the piston seal has freezed to the cylinder, causing an initially, extremely high friction force.
} 


\section{Implementation and Results}

PFC. From (28) and (29) with $\rho=4$, and the choice $n_{f}(s)=1, d_{f}(s)=s+\omega_{f}$, we obtain the PFC

$$
F(s)=\frac{\kappa}{\omega_{f}} \frac{\left(\kappa^{-2} s+1\right)\left(\kappa^{-4} s+1\right)\left(\kappa^{-8} s+1\right)}{\left(\omega_{f}^{-1} s+1\right)(s+1)^{3}}
$$

This simple form lets us easily give the PFC the desired properties by first tuning $\kappa$ to satisfy $\kappa>\kappa_{0}^{+}$. Note that the PFC adds an additional robustness margin to the adaptive system, thus $\kappa$ should not be chosen arbitrary high. A good choice is $\kappa=2 \kappa_{0}^{+}$. Then, we choose $\omega_{f}$ to satisfy $\kappa \omega_{f}^{-1}=0.5 \delta_{0}$, which makes the PFC's effect on tracking performance insignificant. In the case of the clutch actuator, this gives $\kappa=5, \omega_{f}=2500$.

Reference model. The reference model is chosen as

$$
G_{m}(s)=\frac{1}{\left(\tau_{m} s+1\right)^{4}}
$$

with poles at $-1 / \tau_{m}$. The high compressibility and dry friction in the system causes a time delay in the transient response. Thus a higher-order model is preferred over a simple 1st-order model, as its transient response is closer to the properties of the nonlinear system.

Restriction on bandwidth. From simulations on the nonlinear model, with $\delta_{0}=0.1 \mathrm{~mm}$, we find the estimate $\tau_{m \min }=0.5 \mathrm{~s}$. This implies that the achievable bandwidth of the adaptive algorithm without modifications is less than $2 \mathrm{rad} / \mathrm{s}$. Figure 6 shows the adaption of $k(t)$ for a square wave input $r(t)$ with amplitude $10 \mathrm{~mm}$, and high update gain; $\gamma=5 \times 10^{11}$. With $\tau_{m}=0.5(\mathrm{a})$, the fecdback gain $k(t)$ converges to the value 5.4. However, for $\tau_{m}=0.3<\tau_{m \min }$ (b), the system is not able to achieve the specified tracking task for any $k(t)$, thus the algorithm goes unstable.

Peak-cutting. The severe restriction on achievable bandwidth for the adaptive controller is effectively removed by the proposed peak-cutting modification. Figure 6 (c), illustrates the convergence properties of $k(t)$ for $\tau_{m}=0.03 \mathrm{~s}$. Here we have used

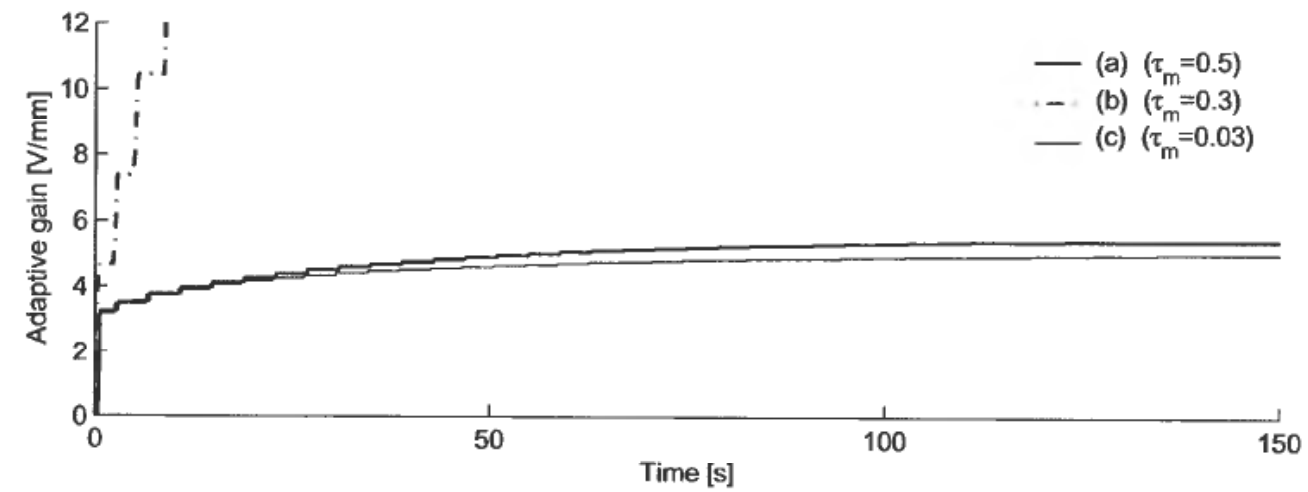

Figure 6. Adaption of $k(t)$ for the unmodified algorithm (a, b), and the modified algorithm (c). The reference input $r(t)$ is a squarc wave, with amplitude $10 \mathrm{~mm}$ and period of $4 \mathrm{~s}$. 

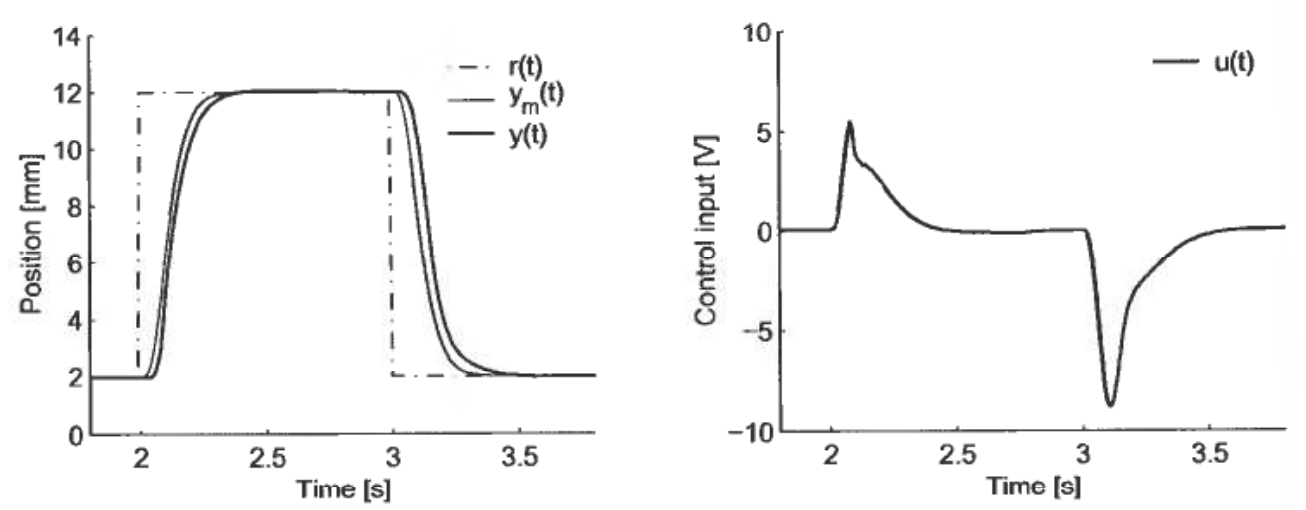

Figure 7. Tracking performance and corresponding control input with adaptive gain $k(t)$ converged to 3.5 .

$\Omega_{m}=\left\{\left.\forall \mathbf{x}_{m}||\left[y_{m}, \tau \dot{y}_{m}, \tau^{2} \ddot{y}_{m}, \tau^{3} y_{m}^{(3)}\right]\right|_{\infty}<\rho_{m}\right\}$, with $\tau=\tau_{m \min }=0.5, \rho_{m}=14.5 \times 10^{-3}$, $\gamma=2 \times 10^{11}$. The adaptive gain eventually converges to 5.1.

The choice $\tau=2 \tau_{m \text { min }}$ gives is a reasonable stability margin for the adaptive system. With this choice, the adaptive gain converges to the limit 3.5. In Figure 7 the tracking performance for $k(t)=3.5$, is illustrated with a step input of height $10 \mathrm{~mm}$, where a normal clutch operation is in the range $2-10 \mathrm{~mm}$. The used control input is within the available $\pm 10 \mathrm{~V}$, and the tracking is achieved with a maximum settling time of $\tau_{s}=150 \mathrm{~ms}$.

Leakage. When including the leakage term (34) in the controller with peak-cutting, the convergence results are qualitatively the same for $k_{+}$higher than 3.5 . With lower $k_{+}$the final value of $k(t)$ is correspondingly reduced.

\section{Conclusions}

The paper illustrates the simplicity in design of the adaptive algorithm, and its application to the electro-pneumatic servo system. The algorithm guarantees boundedness, and convergence of the tracking error to a specified precision, despite the presence of bounded disturbances, and is based on a parallel fecdforward compensator (PFC) to remove the relative-degree-one restriction. A new, practical method for constructing the PFC for arbitrary minimum-phase systems is proposed, where it is worth noting its applicability to relative-degree-one designs of traditional model reference adaptive control (MRAC).

The direct application of the adaptive algorithm has a strong restriction in achievable bandwidth due to the time delay caused by the compressibility in the system. This restriction is effectively removed by allowing adaption for only the lowfrequency content in the reference input. Furthermore, at the expense of a known upper bound on the feedback gain, we introduce a modification that gives additional robustness to disturbances which exceeds the assumed bound. Finally, we show that the modified adaptive algorithm achieve tracking corresponding to a settling-time of $150 \mathrm{~ms}$.

\section{References}

Joannou, P. A. \& DatTA, A. (1991). Robust adaptive control: A unified approach, Proceedings of the IEEE 79(12), 1736-1768. 
KAASA, G.-O. (1999). Nonlinear control of an electropneumatic automotive clutch actuation system, Master's thesis, Norwegian University of Technology and Science, NTNU.

KaAsa, G.-O., Chapple, P. J. \& Lie, B. (2001). An extended kalman filter applied to a pneumatic servo system - velocity and acceleration estimation in a clutch actuation application, in C. R. Burrows \& K. A. EdGE, (Eds). Proceedings, Power Transmission and Motion Control Conference PMTC200 1, Professional Engineering Publishing Ltd., London and Bury St. Edmunds, UK.

Miller, D. E. \& Davidson, E. J. (1991). An adaptive controller which provides an arhitrary good transient and steady-state response, IEEE Thans. Automat. Contr. AC-36(1), 68-81.

Peterson, B. B. \& Narendra, K. S. (1982). Pounded error adaptive control, IEEE Trans. on Autom. Contr. AC-27(6), 1161-1168.

Takahashi, M., Mizumoto, I., Sehorg, D. E. \& Twai Z. (2001). An adaptive tracking control system based on switching compensators, in Proceedings of the 40th IEEE Conference on Decision and Control, Florida, USA.

TANAKA, H. \& WADA, H. (1995). 'Fuzzy control of clutch engagement for automated manual transmissions', Vehicle System Dynamics 24, 365-376. 